\title{
Síndrome de reacción a drogas con eosinofilia y síntomas sistémicos asociado a herpesvirus humano 6. Caso pediátrico tratado con ciclosporina y corticoides
}

\author{
Drug reaction with eosinophilia and systemic symptoms syndrome associated \\ with human herpesvirus 6. A pediatric case treated with cyclosporine and \\ corticosteroids
}

\author{
Dra. Margarita Sánchez Chacón ${ }^{a}$, Dr. Yerco Goldman Pérez ${ }^{a}$, Dra. Stephanie Saavedra Portales ${ }^{a}$ \\ Dr. Javier Arellano ${ }^{a, b}$, Dra. Elena Kakarieka ${ }^{c}$ y Dra. Yamile Corredoira ${ }^{c}$
}

\begin{abstract}
RESUMEN
La reacción a drogas con eosinofilia y síntomas sistémicos es una reacción adversa cutánea rara, potencialmente grave. Puede presentar fiebre, erupción cutánea polimorfa, edema facial y/o linfoadenopatías. La reactivación del virus herpes humano tipo 6 se asocia a un curso más grave y / o prolongado. Un lactante de 22 meses en tratamiento con fenobarbital presentó lesiones eritematopapulares, fiebre, leucocitosis, proteína C reactiva elevada y alteración de pruebas hepáticas. Se realizó biopsia de piel compatible con reacción adversa a drogas. Se trató con corticoides sistémicos e inmunoglobulina intravenosa sin respuesta. La reacción en cadena de la polimerasa para virus herpes humano tipo 6 resultó positiva. Se inició ciclosporina más prednisona, con buena respuesta. Existe poca evidencia del uso de ciclosporina en adultos, cuando los corticoides sistémicos son inefectivos. Este es el primer reporte pediátrico Podría ser una alternativa efectiva o un complemento de los corticosteroides sistémicos cuando no responde a tratamientos convencionales.

Palabras clave: sindrome de hipersensibilidad a medicamentos, herpesvirus humano 6, ciclosporina, pediatría.
\end{abstract}

\begin{abstract}
Drug reaction with eosinophilia and systemic symptoms is a rare and potentially serious skin adverse reaction, with fever, polymorphous skin rash, facial edema, and/or lymphadenopathy. Reactivation of human herpes virus type 6 has been associated with a more severe and/or prolonged course. A 22-month-old infant under phenobarbital treatment developed erythematous-papular lesions, fever, leukocytosis,
\end{abstract}

a. Departamento de Dermatología, Facultad de Medicina, Universidad de Chile, Santiago, Chile.

b. Servicio de Dermatología, Hospital Clínico San Borja de Arriarán, Santiago, Chile.

c. Servicio de Anatomía Patológica, Hospital Clínico San Borja Arriarán, Santiago, Chile.

Correspondencia:

Dra.Margarita Sánchez Chacón:Mar.sanchez.1191@gmail.com

Financiamiento: Ninguno.

Conflicto de intereses: Ninguno que declarar.

Recibido: 2-7-2020

Aceptado: 6-11-2020 elevated C-reactive protein, and abnormal liver tests. The skin biopsy was compatible with an adverse drug reaction. Treatment with systemic corticosteroids and intravenous immunoglobulin had no response. Polymerase chain reaction for human herpesvirus type 6 was positive, and cyclosporine plus prednisone was started with a good response. There is little evidence for the use of cyclosporine in adults when systemic corticosteroids are ineffective. This is the first report of pediatric drug reaction with eosinophilia and systemic symptoms treated with cyclosporine, which could be an effective alternative or an adjunct to systemic corticosteroid therapy unresponsive to conventional treatments.

Key words: drug hypersensitivity syndrome, human herpesvirus 6, cyclosporine, pediatrics.

http: / / dx.doi.org/10.5546/ aap.2021.e247

Cómo citar: Sánchez Chacón M, Goldman Pérez Y, Saavedra Portales S, Arellano J, et al. Síndrome de reacción a drogas con eosinofilia y síntomas sistémicos asociado a herpesvirus humano 6. Caso pediátrico tratado con ciclosporina y corticoides. Arch Argent Pediatr 2021;119(3):e247-e251.

\section{INTRODUCCIÓN}

La reacción a drogas con eosinofilia y síntomas sistémicos (drug reaction with eosinophilia and systemic symptoms; DRESS, por sus siglas en inglés; o drug-induced hypersensitivity; DIHS, por sus siglas en inglés) es una reacción farmacológica adversa cutánea rara pero potencialmente grave, con una tasa de mortalidad de hasta un $10 \% .{ }^{1} \mathrm{El}$ cuadro clásico del síndrome de DRESS comienza con fiebre y, posteriormente, se desarrolla una erupción cutánea polimorfa de larga duración junto con edema facial y linfoadenopatías. En los exámenes de laboratorio, se puede encontrar eosinofilia, linfocitosis atípica y hepatitis. Otras manifestaciones sistémicas menos frecuentes incluyen la carditis, neumonitis, nefritis, entre otras, que le configuran gravedad al estar presentes. $^{2}$ 
A diferencia de otras reacciones adversas a drogas graves, se caracteriza por presentar un largo período de latencia desde la exposición farmacológica (de 2 a 6 semanas). ${ }^{3}$ La reactivación del virus del herpes, especialmente, el virus del herpes humano tipo 6 (VHH-6), a menudo, descrito en el DRESS e incluso considerado un criterio diagnóstico para el DIHS por la literatura japonesa, se ha asociado a un curso más grave y/o prolongado. ${ }^{4,5}$ Se ha reportado en un $36 \%$ de los casos del Registro Europeo de Reacciones Cutáneas Graves Adversas a Medicamentos (European Registry of Severe Cutaneous Adverse Reactions, RegiSCAR). ${ }^{3}$ Sin embargo, en algunas series asiáticas, puede alcanzar hasta un $60 \% .{ }^{5} \mathrm{~A}$ continuación, se presenta un caso pediátrico de un síndrome de DRESS asociado a VHH-6 positivo, tratado con ciclosporina y corticoides.

\section{CASO CLÍNICO}

Lactante de 22 meses de edad, con antecedentes de síndrome bronquial obstructivo crónico, alergia a la proteína de la leche de vaca y síndrome convulsivo. Por este último, se inició, por primera vez, tratamiento con fenobarbital hacía 4 semanas. Presentó la aparición de lesiones eritematopapulares infiltradas con áreas más violáceas de aspecto purpúrico en los hombros, el pliegue inguinal y los muslos, que, posteriormente, se extendieron a la cara, el tronco y las extremidades, no pruriginosas, asociado a linfoadenopatías cervicales y axilares, sin edema ni compromiso de mucosas (Figura 1).

A las 48 horas de evolución, se agregó fiebre hasta $38,5^{\circ} \mathrm{C}$. Debido a esto, consultó al Servicio de Urgencia, donde se sospechó exantema viral, y se tomaron exámenes generales, que destacaron hematocrito: el 44,6 \%; leucocitos: $16050 / \mathrm{ml}$ (eosinófilos: el 1,4 \%); proteína $\mathrm{C}$ reactiva (PCR): $8,9 \mathrm{mg} / \mathrm{l}$; orina completa y radiografía de tórax: normales. Se indicó el manejo sintomático y control ambulatorio. Sin embargo, dado el aumento del exantema y cuadro febril de 5 días de evolución, volvió a consultar en el Servicio de Urgencia, donde se decidió hospitalizar para el estudio y manejo.

Al ingresar, se tomaron exámenes de laboratorio, en los que se destacó hemoglobina: 12,9 g/dl; leucocitos: $19390 / \mathrm{ml}$, sin linfocitos atípicos; eosinófilos: el 1,5\%; velocidad de eritrosedimentación: $5 \mathrm{~mm} / \mathrm{h}$; PCR: 17,5 mg/l; bilirrubina total: $0,3 \mathrm{mg} / \mathrm{dl}$; gamma-glutamil transpeptidasa (GGT): $656 \mathrm{U} / 1$; glutámicooxalacética transaminasa (GOT): $206 \mathrm{U} / 1$; fosfatasa alcalina (FA): $287 \mathrm{U} / 1$.

Se realizaron los siguientes exámenes para evaluar causas infecciosas del exantema: hemocultivos I y II, panel respiratorio por FilmArray, serología de virus de la hepatitis B y C, test para virus de la inmunodeficiencia humana mediante ensayo por inmunoabsorción ligado a enzimas (enzyme-linked immunosorbent assay; ELISA, por sus siglas en inglés), examen de prueba serológica para la sífilis (venereal disease research laboratory; VDRL, por sus siglas en inglés), títulos de antiestreptolisina $\mathrm{O}$, cultivo faríngeo, prueba de Paul-Bunnell, PCR en sangre para virus de Epstein-Barr, parvovirus B19 y citomegalovirus. Todos ellos, negativos.

Además, se realizó un ecocardiograma, que resultó normal, y ferritina, en rangos normales: $104 \mathrm{mg} /$ dl. Fue evaluado por Dermatología, donde se sospechó reacción adversa a medicamentos grave y se decidió realizar una biopsia incisional de piel, que mostró una dermatitis perivascular linfocitaria compatible con

Figura 1. Exantema maculopapular eritematoso, confluente, con zonas de aspecto purpúrico, en la espalda

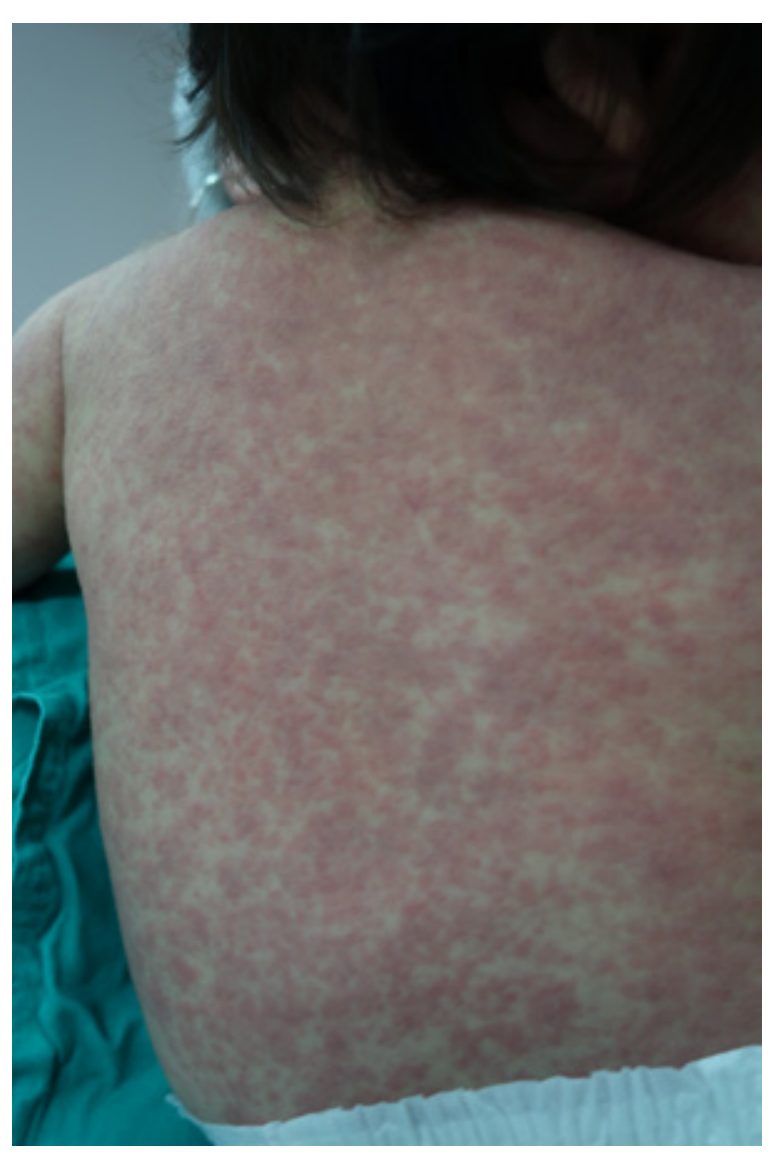


reacción adversa a drogas (Figura 2) y presentó un puntaje RegiSCAR 6/8. Se inició prednisona ( $1 \mathrm{mg} / \mathrm{kg} /$ día). Sin embargo, después de 7 días de tratamiento, el paciente evolucionó eritrodérmico, con un aumento de parámetros inflamatorios y persistencia de alteraciones hepáticas. Se destacaron leucocitos: $40100 / \mathrm{ml}$; eosinófilos: el $5 \%$; GOT: 357 U /1; GPT: 422 U /1; GGT: 600 U/1; FA: $400 \mathrm{U} / \mathrm{l}$; bilirrubina total: $0,6 \mathrm{mg} / \mathrm{dl}$; lactato deshidrogenasa: $2256 \mathrm{UI} / 1$; razón normalizada internacional (international normalized ratio; INR, por sus siglas en inglés): 1,78. Debido a esto, se iniciaron bolos de $30 \mathrm{mg} / \mathrm{kg}$ de metilprednisolona, $1 \mathrm{~g} / \mathrm{kg}$ de inmunoglobulina intravenosa (IGIV) en dos días y, dado el aumento del tiempo de protrombina, se administró vitamina $\mathrm{K}$ parenteral $(0,5 \mathrm{mg} / \mathrm{kg} /$ peso $)$.

Después de 8 días, el paciente evolucionó en malas condiciones generales, somnoliento, con persistencia de fiebre, asociado a ascitis y deterioro de exámenes de laboratorio, a pesar del tratamiento con vitamina $\mathrm{K}$, compatibles con falla hepática aguda. Se observó la presencia de leucocitos: $18300 / \mathrm{ml}$; eosinófilos: el $17 \%$; GOT: 556 U / 1; GPT: 467 U / 1; GGT: 547 U / 1; bilirrubina total: $6,6 \mathrm{mg} / \mathrm{dl}$; tiempo de protrombina: el $31 \%$; INR: 2,6. Se decidió solicitar reacción en cadena de la polimerasa (polymerase chain reaction; $P C R$, por sus siglas en inglés) de VHH-6 en sangre, la cual resultó positiva. Posteriormente, debido al empeoramiento clínico, a pesar del tratamiento con corticoides e inmunoglobulinas, se decidió el inicio de ciclosporina en primera instancia con $5 \mathrm{mg} / \mathrm{kg} /$ día, pero, dados los niveles sanguíneos bajos, se decidió aumentar a $10 \mathrm{mg} / \mathrm{kg} /$ día, con el consentimiento previo de los padres de su uso off-label, asociado a prednisona ( $2 \mathrm{mg} / \mathrm{kg} /$ día), con buena respuesta clínica y de laboratorio a los 5 días de tratamiento.

Finalmente, se dio el alta luego de 30 días de hospitalización, habiendo completado 2 semanas de tratamiento con ciclosporina y prednisona, y manteniéndose la terapia por un mes. Luego, se inició el descenso gradual de ambos tratamientos y se suspendieron a los 2 meses de haber comenzado el descenso sin efectos adversos ni recidiva de lesiones en la piel.

\section{DISCUSIÓN}

El DRESS es una reacción farmacológica grave, con tasas de mortalidad de hasta un $10 \%$, asociada comúnmente a la disfunción hepática (el 51-87 \%), que es la causa más común de muerte. ${ }^{6,7}$ Los medicamentos más frecuentemente asociados

FIgURA 2. Dermatitis de interfase vacuolar linfocitaria y extravasación de eritrocitos con paraqueratosis focal (400X. Hematoxilina y eosina)

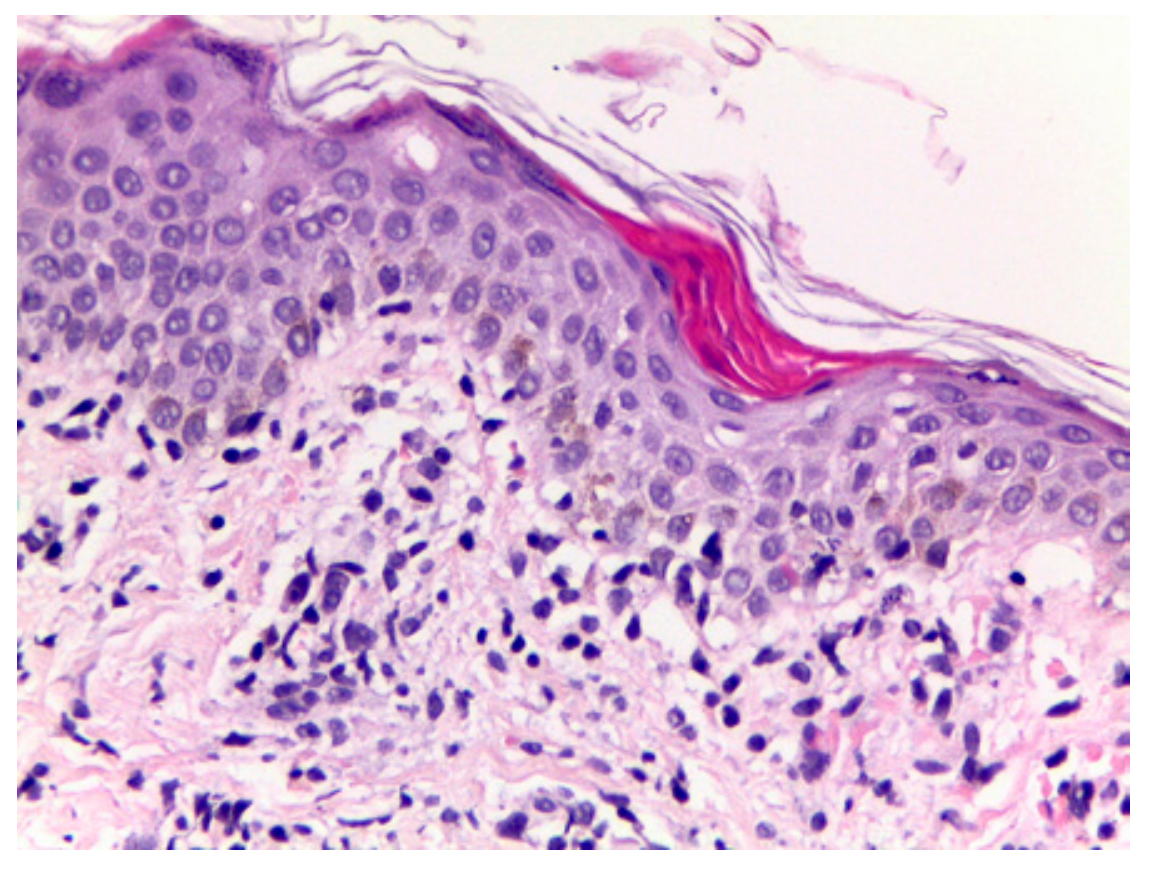


con el síndrome de DRESS en niños incluyen, en especial, los llamados anticonvulsivantes aromáticos (aquellos con una estructura de aminas aromáticas). Estos incluyen fenitoína, fenobarbital, carbamazepina, lamotrigina, felbamato, oxcarbazepina y zonisamida. Una gran cantidad de otras drogas también pueden estar involucradas. Una revisión de 12 años de los casos reportados encontró 44 presuntos fármacos incitantes. ${ }^{8}$ El más común fue la carbamazepina, que representaba, aproximadamente, una cuarta parte de todos los informes de casos.

El DRESS representa una serie de desafíos para el clínico, dado que debe distinguirse de otras reacciones adversas graves, como el síndrome de Stevens-Johnson/necrólisis epidérmica tóxica, pustulosis exantemática aguda generalizada, infecciones virales, síndrome hipereosinofílico, linfoma o enfermedades autoinmunes. Especialmente en la población pediátrica, el síndrome de DRESS comparte características con muchas enfermedades graves, como la enfermedad de Kawasaki, el síndrome de piel escaldada estafilocócica o la artritis inflamatoria juvenil sistémica. ${ }^{9}$ El diagnóstico es clínico, y el manejo incluye el retiro del agente causal y el manejo de soporte. En los casos de afectación de órganos internos, generalmente, se utilizan los corticosteroides sistémicos.

En la población pediátrica, Oh et al., ${ }^{10}$ realizaron una revisión retrospectiva de 47 casos de reacciones adversas a medicamentos graves y concluyeron que 36 pacientes (el $76,6 \%$ ) recibieron esteroides sistémicos; 21 (el 44,7\%), IGIV, y 13 (el $27,7 \%$ ), esteroides sistémicos e IGIV. Se administró ciclosporina solo a un paciente, junto con corticoides sistémicos, y este caso fue un síndrome de Stevens-Johnson.

A pesar de la poca evidencia, basada, generalmente, en reportes de casos clínicos, existen datos del uso exitoso de ciclosporina en pacientes adultos, como terapia inmunosupresora, cuando el tratamiento con corticoides sistémicos no es efectivo, ${ }^{11-13}$ o está contraindicado. Recientemente, Nguyen et al., ${ }^{14}$ realizaron una revisión retrospectiva de 5 casos de DRESS en adultos en los que se utilizó como terapia de primera línea ciclosporina versus el tratamiento con corticoides sistémicos. Concluyeron que el uso de ciclosporina se asociaba a una progresión más corta de la enfermedad, mejores resultados en los marcadores clínicos y de laboratorio en comparación con los esteroides y una menor estadía hospitalaria. Sin embargo, no existen, hasta la fecha, datos publicados de su uso exitoso en la población pediátrica.

En nuestro caso, el paciente representaba un desafío terapéutico debido a la escasa literatura pediátrica y a múltiples marcadores de mal pronóstico, entre ellos: mala respuesta al tratamiento con corticoide e IGIV, falla hepática aguda y reactivación de VHH-6. Sin embargo, en relación con este último, no está claro si la reactivación viral es el evento inicial que induce la expansión de células $\mathrm{T}$ (que reaccionarían de forma cruzada con el fármaco) o si la reactivación es un marcador temprano de la estimulación de las células del sistema inmune (linfocitos T y monocitos / macrófagos). ${ }^{15}$ Son necesarios más estudios para aclarar el papel de los virus en la patogenia de DRESS.

En nuestro paciente, se utilizó la ciclosporina como terapia de tercera línea y con buena respuesta clínica, a pesar de los factores de mal pronóstico. Este es el primer reporte de DRESS pediátrico tratado con ciclosporina, una droga que podría ser una alternativa efectiva o un complemento a la terapia con corticosteroides sistémicos en la población pediátrica que no responde a tratamientos convencionales.

\section{REFERENCIAS}

1. Chen YC, Chiu HC, Chu CY. Drug reaction with eosinophilia and systemic symptoms: a retrospective study of 60 cases. Arch Dermatol. 2010; 146(12):1373-9.

2. Husain Z, Reddy BY, Schwartz RA. DRESS syndrome: part I. Clinical perspectives. J Am Acad Dermatol. 2013; 68(5):693. e1-14.

3. Kardaun SH, Sekula P, Valeyrie-Allanore L, Liss Y, et al. Drug Reaction with Eosinophilia and Systemic Symptoms (DRESS): an original multisystem adverse drug reaction. Results from the prospective RegiSCARstudy. BrJDermatol. 2013; 169(5):1071-80.

4. ShioharaT,InaokaM,KanoY.Drug-inducedhypersensitivity syndrome(DIHS): a reaction induced by a complexinterplay among herpes viruses and antiviral and antidrug immune responses. Allergol Int. 2006; 55(1):1-8.

5. Tohyama M, Hashimoto K, Yasukawa M, Kimura H, et al. Association of human herpesvirus 6 reactivation with the flaring and severity of drug-induced hypersensitivity syndrome. Br J Dermatol. 2007; 157(5):934-40.

6. Chiou CC, Yang LC, Hung SI, Chang YC, et al. Clinicopathological features and prognosis of drug rash with eosinophilia and systemic symptoms: a study of 30 cases in Taiwan. J Eur Acad Dermatol Venereol. 2008; 22(9):1044-9.

7. EshkiM, Allanore L,MusetteP,Milpied B, etal. Twelve-year analysis of severe cases of drug reaction with eosinophilia and systemic symptoms: a cause of unpredictable multiorgan failure. Arch Dermatol. 2009; 145(1):67-72.

8. Camous X, Calbo S, Picard D, Musette Pl. Drug reaction with eosinophilia and systemic symptoms: an update on pathogenesis. Curr Opin Immunol. 2012; 24(6):730-5.

9. Fernando SL. Drug-reaction eosinophilia and systemic symptoms and drug-induced hypersensitivity syndrome. 
Australas J Dermatol. 2014; 55(1):15-23.

10. Oh HL, Kang DY, Kang HR, Kim S, et al. Severe cutaneous adverse reactions in Korean pediatric patients: A study from the Korea SCAR Registry. Allergy Asthma Immunol Res. 2019; 11(2):241 $\square 53$.

11. Zuliani E, Zwahlen H, Gilliet F, Marone C. Vancomycininduced hypersensitivity reaction with acute renal failure: resolution following cyclosporine treatment. Clin Nephrol. 2005; 64(2):155-8.

12. Kirchhof MG, Wong A, Dutz JP. Cyclosporine treatment of drug-induced hypersensitivity syndrome. JAMA Dermatol. 2016; 152(11):1254-7.
13. Harman KE, Morris SD, Higgins EM. Persistent anticonvulsant hypersensitivity syndrome responding to ciclosporin. Clin Exp Dermatol. 2003; 28(4):364-5.

14. Nguyen E, Yanes D, ImadojemuS, Kroshinsky D. Evaluation of Cyclosporine for the Treatment of DRESS Syndrome. JAMA Dermatol. 2020; 156(6):704-6.

15. Roujeau JC, Dupin N. Virus Reactivation in Drug Reaction with Eosinophilia and Systemic Symptoms (Dress) Results from a Strong Drug-Specific Immune Response. J Allergy Clin Immunol Pract. 2017; 5(3):811-2. 